\title{
Comparing perceived heating effect and intensity of therapeutic ultrasound between breast tissue and calf muscle in lactating women: An observational study
}

\section{Lauren Neill}

Curtin University

Elizabeth Eske

Curtin University

Wan Hui Yip

Curtin University

Lalitha Gurram

Curtin University

Beatriz IR de Oliveira

Curtin University

Adelle McArdle

Monash University Department of Physiology and Pharmacology

Melinda Cooper

MMC Physiotherapy

Angela Jacques

Curtin University

Leanda Jane McKenna ( $\sim$ L.McKenna@curtin.edu.au )

Curtin University School of Physiotherapy and Exercise Science https://orcid.org/0000-0003-30078231

Research

Keywords: Breast, lactating, therapeutic ultrasound, calf, inflammatory conditions, mastitis

Posted Date: May 10th, 2020

DOI: https://doi.org/10.21203/rs.3.rs-26696/v1

License: (c) (1) This work is licensed under a Creative Commons Attribution 4.0 International License. Read Full License 


\section{Abstract}

Background Inflammatory conditions of the lactating breast (ICLB) can cause cessation of breastfeeding and treatment for these conditions should be optimised. Therapeutic ultrasound is the most utilised physiotherapy treatment for ICLB. However, there is no evidence that guides physiotherapists on the most appropriate therapeutic ultrasound parameters to treat ICLB, with current ultrasound treatment protocols based on evidence using musculoskeletal tissue. Therefore, it is necessary to determine whether ultrasound interacts with breast and musculoskeletal tissue differently. This study aimed to determine the difference between breast and calf muscle tissue for perception of heating from therapeutic ultrasound; and the therapeutic ultrasound intensity required to perceive warmth in healthy lactating breast tissue.

Methods Fifty adult lactating women who had exclusively breastfed infants aged 6 months or fewer, were recruited from a private physiotherapy clinic in Queensland, Australia. Consent was obtained to participate in this repeated measures within subject descriptive observational study. Therapeutic ultrasound was sequentially applied to the breast and calf; in a randomly assigned order; using a continuous duty cycle of $1 \mathrm{MHz}, 1 \mathrm{Wcm}^{2}$ intensity for an initial period of 5 minutes. Intensity was then increased by $0.1 \mathrm{Wcm}^{2}$ every 30 seconds. The intensity at which participants reported first perceived warmth and then most tolerable warmth for the breast and the calf was recorded.

Results First warmth was perceived at lower intensities in the breast $(Z=-3.637, p<0.001)$, but there was no difference between locations for most tolerable warmth $(Z=-1.165, p=0.244)$. Factors associated with intensity for perception of first warmth were antidepressant use ( $\beta 0.36995 \%$ confidence interval [95\% Cl: 0.103-0.635], $p=0.007$ ) and location ( $\beta 0.286$ [95\% Cl: 0.055-0.516], $p=0.015)$. Body mass index (BMI) was the only factor associated with intensity for most tolerable warmth $(\beta 0.024$ [95\%Cl: 0.004-0.044], $p$ $=0.017)$. The range of intensities required to perceive heating in the breast were $1-2.5 \mathrm{Wcm}^{2}$.

Conclusions As perception of breast and calf warmth was different, therapeutic ultrasound parameters used to treat musculoskeletal conditions may not be appropriate for ICLB. Antidepressant use and BMI influenced heat perception, highlighting the need to screen for these factors prior to therapeutic ultrasound intervention.

\section{Background}

One in every three mothers cease breastfeeding due to pain or infection associated with inflammatory conditions of the lactating breast (ICLB) [1]. The World Health Organization (WHO) recommends that all infants are exclusively breastfed for the first six months of life [2]. Breastfeeding helps to create a strong emotional and psychological bond between mother and baby and assists optimal development, growth and health $[3,4]$. There is compelling evidence to suggest that lactating and breastfeeding for mother and baby are associated with increased health benefits [5]. Mothers who have lactated have a reduced risk of pre-menopausal breast cancer, ovarian cancer, cardiovascular disease, type II diabetes and metabolic syndrome in comparison to those that have not [5]. Health benefits for breastfed infants include a 
reduced incidence of infectious morbidity, reduced risk of childhood obesity, type I and II diabetes, leukaemia and sudden infant death syndrome [5].

Conditions considered an ICLB may include engorgement, blocked milk ducts and mastitis [6]. The cost of ICLB to the Australian and United Kingdom healthcare systems could be as high as \$120 million [7] and $£ 26.8$ million [8] respectively to treat infants with illnesses associated with early cessation of breastfeeding. In addition to the estimated economic impact, ICLB has negative emotional and physical impacts on mothers with young babies. Symptoms associated with ICLB can be disabling, with mothers suffering with mastitis stating "I have never felt worse" [9 p746] and "I felt as if I might die" [9 p746]. Physical symptoms of ICLB may include both local symptoms (breast pain, tenderness, redness and heat) and systemic symptoms (fever, hot sweats, cold shivers, lethargy, fatigue, nausea and vomiting) [9, 10]. By facilitating breastfeeding through appropriate treatments of ICLB, health care costs and the emotional and physical impacts of ICLB could be reduced [11].

The most commonly reported physiotherapy treatment for ICLB is therapeutic ultrasound (TUS) [12]. Two of three studies [13-15] have demonstrated positive results regarding the efficacy of TUS for treatment of ICLB. A case series $(n=25)$ found that $92 \%$ of mothers reported resolution of symptoms associated with blocked ducts following TUS [14]. A cohort study $(n=30)$ indicated that TUS, patient education and manual therapy provided statistically significant decreases in pain, and increased participant breastfeeding efficacy for blocked ducts [13]. Conversely, a randomised controlled trial $(n=109)$ found no difference between TUS and placebo ultrasound for breast engorgement [15]. Although two of these three studies indicate that TUS may be an effective treatment for ICLB, the studies provide little guidance regarding optimal TUS parameters, as a range of TUS parameters were utilised. Consequently, a range of TUS parameters are used to treat ICLB clinically [12], and it is unknown how lactating women respond to differing TUS intensities. Clinicians need further information to guide their parameter selection, in particular intensity, for the treatment of ICLB.

In contrast, studies have shown that TUS is effective for treatment of skeletal muscle tissue injury [1618]. Recent research, including a systematic review $[16,17]$ found that TUS intensities of less than $1.5 \mathrm{Wcm}^{2}$ reduced pain and healing time in tendon, muscle and ligament inflammatory injuries. However, it is not known if TUS parameters for inflammatory injuries in skeletal muscle are applicable to ICLB, due to the differences in tissue physiology. Whilst skeletal muscle has a high protein content, the lactating breast contains highly vascularised lymph, glandular tissue and milk substrates [19].

There have been no studies comparing the use of TUS on breast tissue to skeletal muscle tissue. Understanding how TUS interacts with different tissue types will help clinicians and policy makers to determine if results from muscular studies can be extrapolated to breast tissue. This evidence may ultimately aid in improving ICLB treatment, providing benefits to mothers by alleviating the substantial impact on their physical $[9,10]$ and mental health $[20,21]$. This promotes long term benefits of uninterrupted breastfeeding as recommended by the WHO [2]. 
The dual aims of this current study were to compare the response of breast tissue with skeletal muscle for the perception of heating from TUS whilst accounting for confounding factors; and to determine the TUS intensity for initial perception of warmth and the most tolerable warmth in healthy breast tissue of lactating women.

\section{Methods}

\section{Study Design}

A repeated measures descriptive observational study design was implemented in accordance with the 'STROBE' checklist [22].

\section{Participants and setting}

Fifty women were recruited between June and August 2019 in Mackay, Queensland, using flyers placed at general practitioner and obstetrician clinics, social media and word of mouth. The research was conducted at a private physiotherapy clinic. All participants were screened for eligibility via telephone. The flow of participants through the study is demonstrated in Fig. 1.

Eligible participants had exclusively breastfed for six months or fewer, were aged 18 or older and spoke English. Exclusion criteria included history of ICLB since the birth of their most recent baby, history of breast or calf cancer, past surgery to the breast or calf, or an active skin condition (e.g. eczema) on the breast or calf (Fig. 1).

\section{Outcome measures}

The primary outcome measure was intensity $\left(\mathrm{Wcm}^{2}\right)$ as measured on the TUS machine output display (Chattanooga, model: Intelect Advanced 2772MC). The intensity was recorded when the participant first reported warmth and when the participant reported the most tolerable level of warmth. To ensure accuracy of the intensity value, the TUS machine was commercially calibrated, held within the same room and used only for the purpose of the study. All measurements were recorded by the physiotherapist in the same location.

Breast and calf skin temperature were recorded via infrared thermometer (Omron, model: MC-720) immediately prior to the application of TUS. The calf was selected for comparison, as this site is commonly used in musculoskeletal TUS studies [23]. In previous studies, inserted thermocouples recorded deep tissue temperature, which is not appropriate for lactating breast tissue due to increased risk of infective mastitis [24]. Thermometer values were checked for consistency prior to testing.

Participant age, height (tape measure), weight (calibrated digital scale, Omron: HN-286), number and age of participants' children and history of ICLB during previous lactation was collected. Data on the use of medications, such as antidepressants was collected on all participants, although for 23 participants this was collected retrospectively. 


\section{Intervention}

Eligible participants were instructed to breastfeed their baby or express breast milk, within one hour prior to testing. The breast with the most recent milk removal was used for testing [25].

Two hours prior to testing, ultrasound gel was placed in the testing room. Ambient temperature was fixed at $24^{\circ} \mathrm{C}$, which standardised environment and gel temperature. A standardised script was used to ensure consistency across participants, who also completed a demographic survey using Qualtrics software (Qualtrics, Provo, UT), and provided their consent to participate. Clothing covering the ipsilateral calf was removed and one layer of towelling covered the calf and breast. Participants acclimatised for 30 minutes to allow skin temperature to equilibrate. An online computer program [26] was used to block randomise the order in which participants received breast or calf testing. Non-trial staff prepared sealed opaque envelopes to conceal allocation of order.

Participants were positioned so that the breast rested in a symmetrical conical shape. A hydrogel breast disc was placed over the nipple (Fig. 2), to contain milk flow in case of milk ejection during TUS [25] and prevent gel contacting the nipple.

Participants read a standard warnings and safety summary sheet prior to testing and kept as a guide throughout [27]. Thermal sensitivity testing was conducted on both locations to ensure the participant was not at risk of burns [27]. Only participants who passed this test had TUS applied.

The inferior lateral region of the breast was tested as it has higher levels of glandular tissue and is more likely to be affected by an ICLB [28]. For the calf, TUS was applied posterior to the fibula, in line with the apex of the calf muscles. A template equivalent to twice the area of the TUS transducer head [29] was used to delineate the treatment area (Fig. 3). The transducer head temperature was recorded prior to the breast and calf applications and indicated that no residual heat remained in the transducer head.

After applying $10 \mathrm{ml}$ of gel, the TUS was applied using overlapping linear strokes, with consistent light pressure at a rate of $55 \mathrm{bpm}$ measured via metronome, using a $5 \mathrm{~cm}^{2}$ soundhead. The ultrasound machine was initially set to continuous duty cycle, head warming off, $1 \mathrm{MHz}$ [30-32] at $1 \mathrm{Wcm}^{2}$ intensity for five minutes. This starting intensity was chosen to ensure testing protocol was completed within one hour in order to minimise participant burden. Intensity was increased incrementally by $0.1 \mathrm{Wcm}^{2}$ every 30 seconds. The physiotherapist checked participants comfort and level of warmth throughout the application. During testing, participants were blinded to intensity values. The physiotherapist recorded the intensity at which the participant first reported warmth and again when she reported highest tolerable level of warmth. Testing ceased when the participant reported most tolerable warmth or 10 minutes after the maximum machine intensity of $2.5 \mathrm{Wcm}^{2}$ was reached, even if the participant had not yet reported their most tolerable warmth.

\section{Data Analysis}


A sample of $n=48$ was required to show a $20 \%\left(R^{2}=0.20, f^{2}=0.25\right)$ difference in US intensity between locations in a linear mixed model with 3 covariates (80\% power, $a=0.05)$ ( $G *$ Power V3.1.9.4) [33].

Data were checked for outliers and transcription errors. Normality was examined via histograms, Q/Q plots and measures of central tendency. Descriptive summaries were based on frequency distributions for categorical data, and means and standard deviations or medians, interquartile ranges and ranges for continuous data, depending on normality.

Unadjusted comparisons between locations (breast and calf) for skin temperatures and TUS intensities at which first warmth and most tolerable warmth were perceived, were conducted using non-parametric Wilcoxon signed rank tests.

Data was treated as repeated measures in order to account for within participant correlation between the two locations. Multilevel mixed effects Tobit (censored) regression models with random subject effects were used as the ultrasound intensity range had floor and ceiling effects $\left(1.0 \mathrm{Wcm}^{2}\right.$ and $2.5 \mathrm{Wcm}^{2}$ respectively). Patient factors including skin temperature, prior breastfeeding, antidepressant use and BMI that may have influenced the differences in TUS intensities at which first warmth and most tolerable warmth were perceived between locations were identified in univariable models. The potential confounding factors that were significant $(p<0.10)$ were subsequently entered into multivariable models along with breast or calf location. Residuals were examined graphically to assess model fit. Results were summarised using Beta coefficients and 95\% confidence intervals.

Data analysis was blinded, hypothesis tests were 2-sided, and $P$ values of $<.05$ were considered statistically significant. Stata version 16.0 (StataCorp LLC College Station, TX) was used for data analysis.

\section{Results}

Fifty women participated in the study; one participant experienced anxiety and testing was terminated and her data removed from the data set (Fig. 1). Most participants had two children and had previously lactated (Table 1). All 49 participants passed thermal sensitivity testing and prior to testing, skin temperature of the breast was warmer than the calf (Table 2). Seven and six participants did not achieve most tolerable warmth in the breast and calf respectively, as the TUS machine was limited to $2.5 \mathrm{Wcm}{ }^{2}$. Testing did not elicit milk flow from the nipple and no adverse events occurred.

\section{Table 1}

Participants' Characteristics $(n=49)$ 


\begin{tabular}{|lll|}
\hline Characteristics & & $\boldsymbol{n}(\%)$ \\
\hline Age (mean (SD)) & & $31.6(4.2)$ \\
\hline BMl (kg/cm2) (mean (SD)) & $26.5(5.1)$ \\
\hline Parity & 1 & $14(28.6 \%)$ \\
\hline & 2 & $23(46.9 \%)$ \\
\hline First experience of breastfeeding & Yes & $8(16.3 \%)$ \\
\hline & No & $34(8.2 \%)$ \\
\hline Antidepressant use & Yes & $3(6.1 \%)$ \\
\hline & No & $46(93.9 \%)$ \\
\hline
\end{tabular}

SD, standard deviation. BMI, body mass index

Table 2

Unadjusted differences in TUS intensity between breast and calf $(n=49)$

\begin{tabular}{|llll|}
\hline & $\begin{array}{l}\text { Breast } \\
\text { Med (IQR) [min-max] }\end{array}$ & $\begin{array}{l}\text { Calf } \\
\text { Med (IQR) [min-max] }\end{array}$ & p \\
\hline Skin temperature $\left({ }^{\circ} \mathrm{C}\right)$ & $33.8(33.1,34.4)[30.4-$ & $30.3(29.8,30.9)[28.5-$ & $<$ \\
& $35.1]$ & $32.6]$ & 0.001 \\
\hline $\begin{array}{l}\text { Intensity 1st perception } \\
\left(\text { Wcm }^{2}\right)\end{array}$ & $1.3(1.1,1.5)[1.0-2.0]$ & $1.5(1.3,1.7)[1.0-2.3]$ & $<$ \\
\hline $\begin{array}{l}\text { Intensity most tolerable } \\
(\text { Wcm }\end{array}$ & $2.2(1.8,2.5)[1.2-2.5]$ & $2.2(1.9,2.5)[1.4-2.5]$ & 0.001 \\
\hline IQR, interquartile range & & & 0.224 \\
\hline
\end{tabular}

Participants perceived first warmth at a lower intensity in the breast as compared to the calf, while perception of most tolerable warmth was not different between locations (Table 2).

With respect to first perception of warmth, the variables that were independently associated with intensity were location, skin temperature, antidepressant use and order of testing (Table 3). Univariate analysis demonstrated that higher initial skin temperature and the site tested subsequently, were associated with a lower intensity for the perception of first warmth. Antidepressant use was independently associated with a higher intensity for first warmth. 
After adjustment for skin temperature, antidepressant use and order of testing, only location and antidepressant use remained significantly associated with ultrasound intensity (Table 3).

\section{Table 3}

Analysis for first perception of warmth $(n=49)$

\begin{tabular}{|llllll|}
\hline & Univariable Models & & Multivariable Model & \\
& $\beta$ Coefficient $(95 \% \mathrm{Cl})$ & $\mathrm{p}$ & $\beta$ Coefficient $(95 \% \mathrm{Cl})$ & $\mathrm{p}$ \\
\hline Location & $0.233(0.125$ to 0.342$)$ & $<0.001$ & $0.286(0.055$ to 0.516$)$ & 0.015 \\
\hline Skin Temperature & $-0.054(-0.085$ to -0.023$)$ & 0.001 & $0.024(-0.042$ to 0.085$)$ & 0.510 \\
\hline Antidepressant use & $0.374(0.010$ to 0.648$)$ & 0.007 & $0.369(0.103$ to 0.635$)$ & 0.007 \\
\hline Order of testing & $-0.145(-0.265$ to -0.024$)$ & 0.018 & $-0.117(-0.223$ to -0.012$)$ & 0.029 \\
\hline BMl & $0.001(-0.014$ to 0.015$)$ & 0.938 & - & - \\
\hline BMI, body mass index; Cl, confidence interval & & & \\
\hline
\end{tabular}

Regarding the most tolerable perceived warmth, body mass index (BMI) was the only variable independently associated with the TUS intensity (Table 4). Order of testing and BMI were entered into the multivariable model with location. Only BMI remained associated with ultrasound intensity after adjustment (Table 4).

\section{Table 4}

Analysis for perception of most tolerable warmth $(n=49)$

\begin{tabular}{|lllll|}
\hline & Univariable Models & & Multivariable Models & \\
\hline & $\beta$ Coefficient $(95 \% \mathrm{Cl})$ & $\mathrm{p}$ & $\beta$ Coefficient $(95 \% \mathrm{Cl})$ & $\mathrm{p}$ \\
\hline BMI & $0.024(0.004$ to 0.044$)$ & 0.016 & $0.024(0.004$ to 0.044$)$ & 0.017 \\
\hline Order of testing & $-0.171(-0.346$ to 0.005$)$ & 0.056 & $-0.156(-0.333$ to 0.0177$)$ & 0.078 \\
\hline Location & $0.110(-0.069$ to 0.288$)$ & 0.229 & $0.088(-0.087$ to 0.263$)$ & 0.326 \\
\hline Skin temperature & $-0.026(-0.075$ to 0.023$)$ & 0.296 & - & - \\
\hline Antidepressant & $0.047(-0.373$ to 0.467$)$ & 0.827 & - & - \\
\hline BMI, body mass index; Cl, confidence interval & & & \\
\hline
\end{tabular}

With respect to the second aim there was a wide variety of intensities that participants reported for first and most tolerable warmth in the breast (Table 2). 


\section{Discussion}

Participants perceived first warmth at a lower intensity in the breast compared to the calf, but there was no difference between locations for most tolerable warmth. After adjusting for confounding factors, these relationships remained. There was a wide range of intensities reported by participants for first perceived and most tolerable warmth.

In relation to the primary aim, differences found between locations for first perceived warmth, may be due to greater tissue heterogeneity and acoustic impedance in breast tissue or psychological and environmental factors [34]. Greater heterogeneity potentially causes greater deflection and absorption of US waves, with subsequent increased superficial heating, and earlier perception of first warmth [35]. Additionally, the difference between the breast and calf may also be due to higher density of cutaneous thermoreceptors in the breast, resulting in an increased sensitivity to the heating [36]. These findings may indicate that choosing TUS parameters for the treatment of ICLB based on TUS parameters used previously in musculoskeletal studies may not be appropriate.

No difference in intensity for most tolerable warmth was found between breast and calf, which could be explained by the upper limit in ultrasound machine intensity. As more than a quarter of participants did not achieve most tolerable warmth, this limits the conclusions that can be drawn from the most tolerable warmth data. A difference between the breast and the calf may have been evident, if ultrasound machines capable of intensities greater than $2.5 \mathrm{Wcm}^{2}$ were available.

In relation to the secondary aim, the perceived range of intensities observed by participants for both first warmth and most tolerable warmth were representative of the entire range of intensities tested from 1.0$2.5 \mathrm{Wcm}^{2}$ (Table 2). This reflects the intensities utilised in clinical practice of $0.8-2.4 \mathrm{Wcm}^{2}$ [12]. As participants perceived heating over a wide range, clinicians may need to continue to provide individualised intensities when applying TUS to the breast, rather than a predetermined set intensity level.

Antidepressant use was associated with a higher intensity for first perceived warmth, indicating that participants who use antidepressants required higher intensities of TUS. Antidepressants supress the body's thermoregulatory system causing decreased peripheral receptor sensitivity [37]. This could explain why antidepressants were perhaps not a significant factor for the perception of most tolerable warmth; therefore these women may need greater intensities for clinical efficacy. When physiotherapists are using TUS to treat women who use antidepressants, additional care is required, as their perception of heating is lower than women who do not take antidepressants.

Participants with a higher BMI were able to tolerate higher intensities of TUS. This could be due to higher fat deposition in the breast compared to the calf [38] resulting in greater dispersion and reduced absorption of ultrasound. Similar to antidepressants, the altered perceived heating effects in those with a high BMI, highlights the need for physiotherapists to monitor this group of patients more carefully. Considering these findings, physiotherapists should consider screening for antidepressant use and calculating BMI in all patients requiring TUS treatment. 
The TUS machine utilised in this study is representative of current machines available in Australia, delivering a maximum intensity of $2.5 \mathrm{Wcm}^{2}$. Physiotherapists in the eastern states of Australia aim to achieve thermal effects of TUS to treat ICLB [12]. However, machines limited to $2.5 \mathrm{Wcm}^{2}$ may not provide the desired perceivable heating effects. Historically, machines were able to deliver a maximum intensity of $3 \mathrm{Wcm}^{2}$ but this was lowered to $2.5 \mathrm{Wcm}^{2}$ following reported heat injuries in musculoskeletal tissue [39]. The current data indicates that the manufactured machine intensity level could be raised to allow physiotherapists to treat ICLB with their desired intensity level. This would facilitate research to determine what range of TUS intensities is effective for treatment of ICLB.

\section{Limitations and strengths}

This study was a perception-based study as opposed to a study looking at breast tissue temperature. Temperature perception can be affected by physiological, psychological and environmental factors, [34] and participants may perceive heating effects of TUS differently. Perception may be influenced by participants' pre-conceived ideas that the breast would be more sensitive than the calf [34]. There have been no studies investigating if perception of warmth accurately reflects change in tissue temperature. Therefore, limited conclusions regarding tissue temperature or heating effects within the tissue can be made. This study was conducted on participants with healthy lactating breast tissue; hence, caution should be applied when extrapolating findings of this study to women with ICLB.

A strength of this study is that it is the first observational study to provide a comparison of perceived heating effects of TUS between lactating breast tissue and skeletal muscle and to empirically determine average intensities that lactating women would perceive heating. Thus, it provides an initial indication that research findings from musculoskeletal studies on the use of TUS may not be extrapolated to the breast.

\section{Future research}

Future studies need to explore alternative ways to assess breast tissue temperature accurately without inserting thermocouples into the breast [24]. Research is needed to determine whether perception of warmth is closely correlated with change in tissue temperature. Research is also needed to determine whether thermal or non-thermal effects of TUS are more effective for ICLB and what parameters should be used.

\section{Conclusion}

This research suggests that the breast and calf do not respond to TUS in the same way, as warmth from TUS was perceived at lower intensities in the breast compared to the calf in lactating women. Therapeutic ultrasound parameters examined in musculoskeletal research and used clinically, may not be appropriate for ICLB. 
The wide range of intensities at which participants perceived warmth was influenced by antidepressant use and BMI. Whilst skin testing prior to indicating TUS treatment is necessary for all patients, physiotherapists need to specifically screen for anti-depressant use and BMI in their clinical interview.

Furthermore, not all participants in this study felt most tolerable warmth at the maximal machine intensity. As a result, manufacturers should consider producing TUS machines with higher intensities, which will enable research to fully investigate TUS treatments for inflammatory conditions of the lactating breast.

\section{Abbreviations}

BMI

Body mass index

$\mathrm{Cl}$

Confidence interval

ICLB

Inflammatory conditions of the lactating breast

IQR

Interquartile range

SD

Standard deviation

TUS

Therapeutic ultrasound

WHO

World Health Organisation

\section{Declarations}

\section{Ethics approval and consent to participate}

Ethics approval was provided by the Human Research Ethics Committee (Approval number: HRE20190382). Consent was obtained by all participants through the use of Qualtrics software.

\section{Consent for publication}

Not applicable

\section{Availability of data and materials}


The datasets used and/or analysed during the current study are available from the corresponding author on reasonable request

\section{Competing interests}

The authors declare that they have no competing interests

\section{Funding}

This research did not receive any specific grant from funding agencies in the public, commercial, or notfor-profit sectors.

\section{Authors' contributions}

LN collected the data. WHY and AJ completed data analysis. AM, LM and MC conceptualised the study. All authors had a major contribution to the writing of the manuscript, read and approved the final manuscript.

\section{Acknowledgements}

We wish to thank all participants, Richard Wright, Physio Plus in Mackay and Dr. Leo Ng for making this research possible.

\section{References}

1. Schwartz K, D'Arcy HJS, Gillespie B, Bobo J, Longeway M, Foxman B. Factors associated with weaning in the first 3 months postpartum. J Fam Pract. 2002;51(5):439-44.

2. World Health Organization. Exclusive breastfeeding for six months best for babies everywhere. [Internet]. 2011 [cited 27 April]. Available from: https://www.who.int/mediacentre/news/statements/2011/breastfeeding_20110115/en/.

3. Mangesi L, Zakarija-Grkovic I. Treatments for breast engorgement during lactation. Cochrane Database Syst Rev. 2016; (6):1-59. doi:10.1002/14651858.CD006946.pub3.

4. Amir LH, Livingstone VH. Management of common lactation and breastfeeding problems. In: Jatoi I, Rody A, editors. Management of breast diseases. Cham: Springer; 2016. 
5. Stuebe AM, Schwarz EB. The risks and benefits of infant feeding practices for women and their children. J Perinatol. 2010;30(3):155-62. doi:10.1038/jp.2009.107.

6. Amir LH. ABM clinical protocol \#4: Mastitis, revised March 2014. Breastfeed Med. 2014;9(5):239-43. doi:10.1089/bfm.2014.9984.

7. Smith JP, Thompson JF, Ellwood A. Hospital system costs of artificial infant feeding: estimates for the Australian Capital Territory. Aust N Z J Public Health. 2002;26(6):543-51. doi:10.1111/j.1467842X.2002.tb00364.X.

8. Renfrew M, Pokhrel S, Quigley M, McCormick F, Fox-Rushby J, Dodds R, et al. Preventing disease and saving resources: the potential contribution of increasing breastfeeding rates in the UK. UK: UNICEF; 2012.

9. Lumley J, Amir LH. Women's experience of lactational mastitis: 'I have never felt worse'. Aust Fam Physician. 2006;35(9):745-747. Available from: <https://search-informit-comau.dbgw.lis.curtin.edu.au/documentSummary; $d n=362082565293497 ;$;res=IELHEA>

10. Lawrence RA, Lawrence RM. Breastfeeding: A guide for the medical professional. 7 ed: Elsevier Health Science; 2010.1128 p. p. 1-1128.

11. House of Representatives, Standing Committee of Health and Ageing. The Best Start: Report on the inquiry into the health benefits of breastfeeding. Canberra: Commonwealth of Australia; 2007. Available from: .

12. Diepeveen L, Fraser E, Croft A, Jacques A, McMrdle A, Briffa K, et al. Regional and facility differences in interventions for mastitis by Australian physiotherapists. J Hum Lact. 2018;35(4):695-705. doi:10.1177/0890334418812041.

13. Cooper BB, Kowalsky D. Physical therapy intervention for treatment of blocked milk ducts in lactating women. JWHPT. 2015;39(3):115-26. doi:10.1097/jwh.0000000000000037.

14. Lavigne V, Gleberzon BJ. Ultrasound as a treatment of mammary blocked duct among 25 postpartum lactating women: a retrospective case series. J Chiropr Med. 2012;11(3):170-8. doi:10.1016/j.jcm.2012.05.011.

15. McLachlan Z, Milne EJ, Lumley J, Walker BL. Ultrasound treatment for breast engorgement: A randomised double blind trial. Aust J Physiother. 1991;37(1):23-8. doi:10.1016/s00049514(14)60531-6.

16. Best TM, Wilk KE, Moorman CT, Draper DO. Low intensity ultrasound for promoting soft tissue healing: A systematic review of the literature and medical technology. Intern Med J. 2016;2(11):1-9. doi:10.18103/imr.v2i11.271.

17. Lewis GK, Langer MD, Henderson CR, Ortiz R. Design and evaluation of a wearable self-applied therapeutic ultrasound device for chronic myofascial pain. Ultrasound Med Biol. 2013;39(8):142939. doi:10.1016/j.ultrasmedbio.2013.03.007.

18. Aaron SE, Delgado-Diaz DC, Kostek MC. Continuous ultrasound decreases pain perception and increases pain threshold in damaged skeletal muscle. Clin J Sport Med. 2017;27(3):271-7. doi:10.1097/jsm.0000000000000343. 
19. Dyson M. Mechanisms involved in therapeutic ultrasound. Physiotherapy. 1987;73:116-20.

20. Brown A, Rance J, Bennett P. Understanding the relationship between breastfeeding and postnatal depression: the role of pain and physical difficulties. J Adv Nurs. 2016;72(2):273-82. doi:10.1111/jan.12832.

21. Madlala SS, Kassier SM. Antenatal and postpartum depression: effects on infant and young child health and feeding practices. South Afr J Clin Nutr. 2017;31(1):1-7. doi:10.1080/16070658.2017.1333753.

22. von Elm E, Altman DG, Egger M, Pocock SJ, Gotzsche PC, Vandenbroucke JP, et al. The Strengthening the reporting of observational studies in epidemiology (STROBE) statement: guidelines for reporting observational studies. Int J Surg. 2014;12(12):1495-9. doi:10.1016/j.ijsu.2014.07.013.

23. Hayes BT, Merrick MA, Sandrey MA, Cordova ML. Three-MHz ultrasound heats deeper into the tissues than originally theorized. J Athl Train. 2004;39(3):230-4.

24. Rivens I, Shaw A, Civale J, Morris H. Treatment monitoring and thermometry for therapeutic focused ultrasound. Int J Hyperthermia. 2009;23(2):121-39. doi:10.1080/02656730701207842.

25. Pang WW, Hartmann PE. Initiation of human lactation: secretory differentiation and secretory activation. J Mammary Gland Biol Neoplasia. 2007;12(4):211-21. doi:10.1007/s10911-007-9054-4.

26. Haahr M. Random.org [Internet] [Internet]. 1998 [cited 28 April]. Available from: https://www.random.org/.

27. Robertson VJ, Chipchase LS, Laakso EL, Whelan KM, McKenna LJ. Guidelines for the clinical use of electrophysical agents St Kilda Road Central. Victoria: Australian Physiotherapy Association; 2001.

28. Geddes DT. Inside the lactating breast: the latest anatomy research. J Midwifery Womens Health. 2007;52(6):556-63. doi:10.1016/j.jmwh.2007.05.004.

29. Demchak TJ, Stone MB. Effectiveness of clinical ultrasound parameters on changing intramuscular temperature. J Sport Rehabil. 2008;17(3):220-9.

30. Draper DO, Schulthies S, Sorvisto P, Hautala AM. Temperature changes in deep muscles of humans during ice and ultrasound therapies: an in-vivo study. J Orthop Sports Phys Ther. 1995;21(3):153-8.

31. Hauck YL, Fenwick J, Dhaliwal SS, Butt J. A Western Australian survey of breastfeeding initiation, prevalence and early cessation patterns. Matern Child Health J. 2011;15(2):260-8. doi:https://doi.org/10.1007/s10995-009-0554-2.

32. Baker KG, Robertson VJ, Duck F. A review of therapeutic ultrasound: biophysical effects. Phys Ther. 2001;81(7):1351-8.

33. Faul F, Erdfelder E, Buchner A, Lang A-G. Statistical power analyses using G*Power 3.1: Tests for correlation and regression analyses. Behav Res Methods. 2009;41(4):1149-60.

34. Schweiker M, Huebner GM, Kingma BRM, Kramer R, Pallubinsky H. Drivers of diversity in human thermal perception: a review for holistic comfort models. Temperature. 2018;5(4):308-42. doi:10.1080/23328940.2018.1534490. 
35. Starkey C. Deep Heating Agents. In: Starkey C, editor. Therapeutic Modalities. Ausgabe: F A Davis Co; 2013. p. 448.

36. Gerrett N, Ouzzahra Y, Havenith G. Distribution of skin thermal sensitivity. In: Humbert P, Fanian F, Maibach H, Agache P, editors. Agache's Measuring the Skin. Cham: Springer; 2017.

37. Mico J, Ardid D, Berrocoso E, Eschalier A. Antidepressants and pain. Trends Pharmacol Sci. 2006;27(7):348-54. doi:10.1016/j.tips.2006.05.004.

38. Wallner SJ, Luschnigg N, Schnedl WJ, Lahousen T, Sudi K, Crailsheim K, et al. Body fat distribution of overweight females with a history of weight cycling. Int J Obes Relat Metab Disord. 2004;28(9):1143-8. doi:10.1038/sj.ijo.0802736.

39. Government of Canada. Guidelines for the Safe Use of Ultrasound: Part II - Industrial \& Commercial Applications - Safety Code 24 Ottawa, Canada:1991.

\section{Figures}

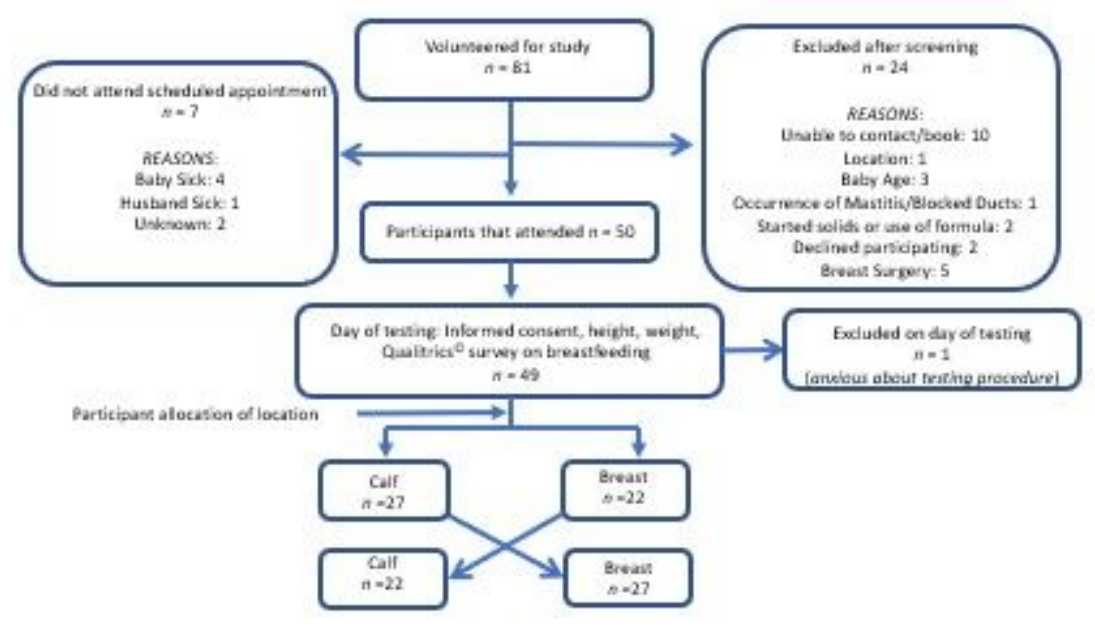

\section{Figure 1}

Flow of participants and methods 


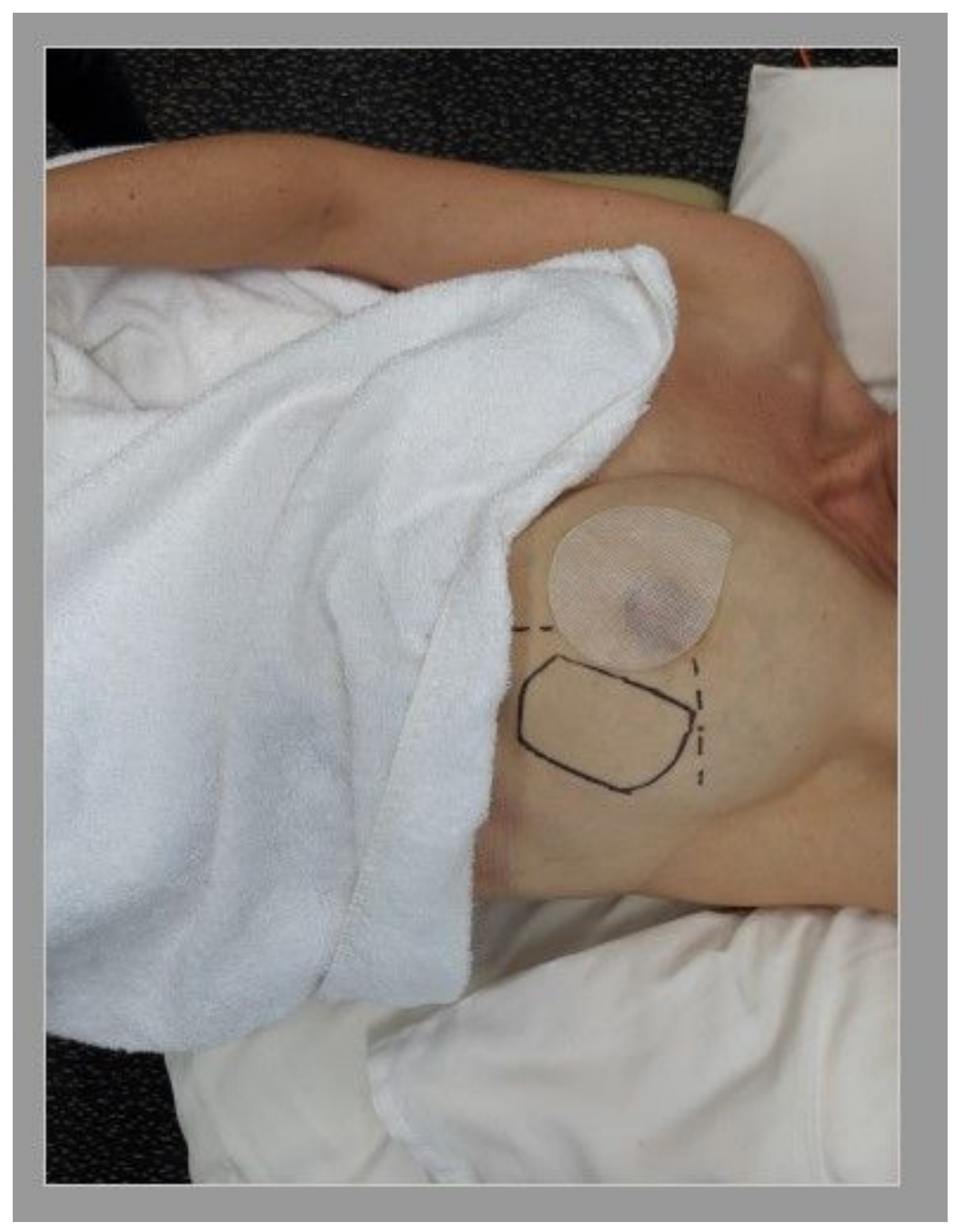

Figure 2

Positioning and area of breast testing 


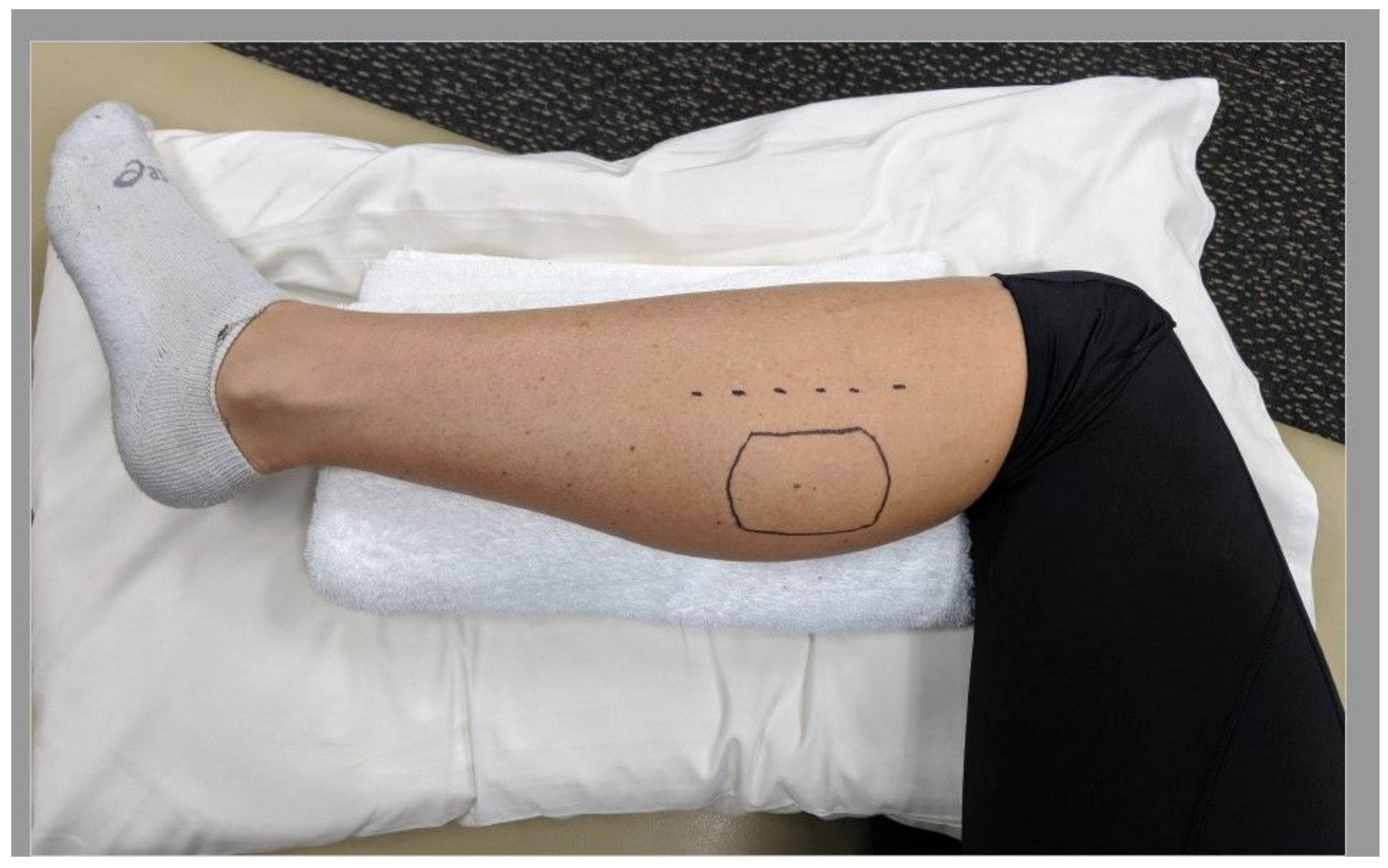

Figure 3

Positioning and area of calf testing 